\title{
Autonomic neuropathy predisposes to rosiglitazone-induced vascular leakage in insulin-treated patients with type 2 diabetes: a randomised, controlled trial on thiazolidinedione-induced vascular leakage
}

\author{
A. J. Rennings • P. Smits • M. W. Stewart • C. J. Tack
}

Received: 21 February 2010 /Accepted: 16 April 2010 /Published online: 25 May 2010

(C) The Author(s) 2010. This article is published with open access at Springerlink.com

\begin{abstract}
Aims/hypothesis The mechanism of fluid-related complications caused by thiazolidinedione derivatives is unclear. One potential mechanism is thiazolidinedione-induced arterial vasodilatation, which results in vascular leakage and a fall in blood pressure, normally counterbalanced by sympathetic activation and subsequent renal fluid retention. We hypothesised that thiazolidinedione-induced vascular leakage will be particularly prominent in patients with autonomic neuropathy. Methods We conducted a randomised, double-blind, placebo-controlled, parallel study in 40 patients with type 2 diabetes on insulin treatment recruited from a university medical centre. The randomisation was performed by a central office using a randomisation schedule. Both treatment groups, placebo $(n=21)$ and rosiglitazone $(n=19)$, were stratified for sex and level of autonomic neuropathy as assessed by Ewing score $(<2.5$ or $\geq 2.5)$. We investigated the effects of 16 weeks of treatment with rosiglitazone $4 \mathrm{mg}$ twice daily on vascular leakage (transcapillary escape rate of albumin, TERalb), body weight, extracellular volume and plasma volume.
\end{abstract}

\footnotetext{
A. J. Rennings $(\bowtie) \cdot$ P. Smits

Department of Pharmacology-Toxicology 149,

Radboud University Nijmegen Medical Centre,

PO Box 9101, 6500 HB Nijmegen, the Netherlands

e-mail: A.Rennings@pharmtox.umcn.nl

P. Smits $\cdot$ C. J. Tack

Department of Internal Medicine,

Radboud University Nijmegen Medical Centre,

Nijmegen, the Netherlands

M. W. Stewart

GlaxoSmithKline,

King of Prussia, PA, USA
}

Results Thirty-nine patients were included in the analysis. In patients with high Ewing scores $(n=16)$, rosiglitazone increased TERalb significantly ( $\triangle$ TERalb: rosiglitazone $+2.43 \pm 0.45 \% / \mathrm{h}$, placebo $-0.11 \pm 0.15 \% / \mathrm{h}, p=0.002$ ), while rosiglitazone had no effect in the patients with low Ewing scores $(n=23)$. Rosiglitazone-induced increases in TERalb and Ewing score at baseline were correlated $(r=0.65$, $p=0.02$ ). There was no correlation between Ewing score and rosiglitazone-induced changes in fluid variables. One subject was withdrawn from the study because of atrial fibrillation.

Conclusions/interpretation Rosiglitazone may increase vascular leakage in insulin-treated patients with type 2 diabetes with autonomic neuropathy. Autonomic neuropathy did not exaggerate rosiglitazone-induced fluid retention. Therefore, autonomic neuropathy should be considered as a risk factor for thiazolidinedione-induced oedema, not for thiazolidinedione-induced fluid retention.

Trial registration ClinicalTrials.gov NCT00422955 Funding GlaxoSmithKline

Keywords Autonomic neuropathy - Clinical science Diabetes mellitus · Human - Oedema - Oral pharmacological agents $\cdot$ Randomised controlled trial $\cdot$ Risk factors .

Thiazolidinediones
Abbreviations
ANP Atrial natriuretic peptide
DBP Diastolic blood pressure
ECV Extracellular volume
PPAR $\gamma$ Peroxisome proliferator activated receptor $\gamma$
SBP Systolic blood pressure
TBW Total body water
TERalb Transcapillary escape rate of albumin 


\section{Introduction}

Thiazolidinedione derivatives are used in the treatment of type 2 diabetes as they improve insulin sensitivity and reduce blood glucose concentration [1,2]. Besides their effect on glycaemia, thiazolidinediones appear to have favourable effects on plasma lipids, blood pressure, fibrinolysis and inflammation, which might offer additional beneficial effects beyond glucose lowering with respect to the prevention of cardiovascular disease [3]. There is intense scientific dispute about whether thiazolidinediones, particularly rosiglitazone, protect against cardiovascular disease or may even increase the risk of ischaemic cardiovascular events [4-7]. Part of the beneficial effects of thiazolidinediones may be outbalanced by side effects, especially fluid retention. For example, the PROspective pioglitAzone Clinical Trial In macroVascular Events (PROActive) study showed a trend towards a reduced risk of cardiovascular events with pioglitazone compared with placebo in persons with type 2 diabetes, but this benefit was largely offset by fluid-related adverse events, oedema formation and heart failure [8]. The mechanism of fluid retention is unclear [9], but unravelling the mechanism would identify risk factors for the use of thiazolidinediones and enable the prescription of thiazolidinedione therapy only for patients with a favourable benefit/risk ratio.

Based on the demonstration in preclinical studies that thiazolidinediones stimulate epithelial sodium channels in the renal collecting duct $[10,11]$, it has been suggested that thiazolidinedione-related fluid retention in humans is caused by primary renal mechanisms, although a recent preclinical study showed opposing results [12] and human experimental data are lacking. Primary renal sodium retention would also increase blood pressure, which is at odds with the observed blood pressure lowering effect of thiazolidinediones [13, 14]. As the initiating key mechanism, thiazolidinedione-induced arterial vasodilatation [9] explains both blood pressure reduction and fluid retention. The local microvascular consequence of arterial vasodilatation is increased hydrostatic capillary pressure with more vascular leakage and the formation of interstitial fluid. The systemic consequence is reduction in peripheral vascular resistance [15] and blood pressure, which is the driving force for secondary renal sodium retention. Meanwhile, the sympathetic nervous system counteracts the vasodilator effect [16], reducing the increment in vascular leakage and preventing an exaggerated fall in blood pressure and the consecutive increment in sodium retention. Sympathetic activation also directly stimulates renin production, which induces sodium retention. Therefore, the net effect of the increased sympathetic tone on renal sodium retention is unclear (Fig. 1).

Patients with autonomic failure are not able to counterbalance haemodynamic changes effectively, which will result in an unopposed change in capillary hydrostatic force. Indeed, some case reports suggest that the haemodynamic effects of insulin are exaggerated in persons with autonomic neuropathy [17, 18], while blockade of the autonomic nervous system may increase insulin-induced vascular leakage [19].

Epidemiological data support this mechanistic line of reasoning. It has been reported that the incidence of oedema

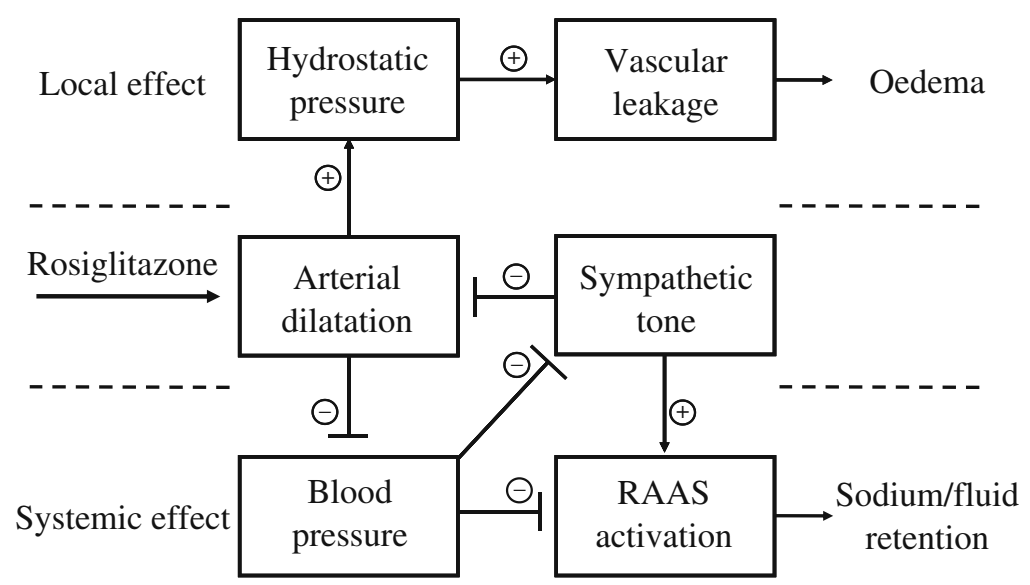

Fig. 1 Schematic representation of the hypothesis. The local consequence of rosiglitazone-induced vasodilatation will be increased hydrostatic pressure leading to an elevation in capillary filtration (vascular leakage), which predisposes to oedema formation (top). An intact sympathetic nervous system counteracts the vasodilator effect, which will prevent increased vascular leakage. The systemic consequence of vasodilatation is reduction of blood pressure (bottom), leading to renin production and sodium retention. On the one hand, an intact sympathetic nervous system prevents reduction in blood pressure immediately; on the other hand, sympathetic activation directly stimulates renin production; the result is diminished and elevated sodium retention respectively. RAAS, renin-angiotensinaldosterone system 
is higher when thiazolidinediones are combined with insulin [9]. This may be due to a combined effect of thiazolidinediones and insulin or to complications accompanying longstanding diabetes [9]. In a previous study in insulin-resistant people without diabetes, we found no evidence for adverse vascular effects of the combined use of rosiglitazone and insulin [20], suggesting that it is not insulin itself but probably complications associated with longstanding diabetes that render patients prone to oedema formation. Autonomic neuropathy is a typical complication of longstanding diabetes $[21,22]$.

In the present study in insulin-treated patients with type 2 diabetes, we investigated whether thiazolidinedioneinduced microvascular leakage is more pronounced in patients with autonomic neuropathy. We also investigated whether autonomic neuropathy affects thiazolidinedioneinduced fluid retention.

\section{Methods}

The study population consisted of 40 participants with type 2 diabetes, who had received insulin treatment for at least 6 months. Further inclusion criteria were age between 30 and 75 years, body mass index $<40 \mathrm{~kg} / \mathrm{m}^{2}$ and fasting plasma glucose between 7.0 and $15 \mathrm{mmol} / \mathrm{l}$. Participants were not eligible for inclusion if $\mathrm{HbA}_{1 \mathrm{c}}$ was higher than $12 \%$, if they used over $200 \mathrm{U}$ insulin a day, if they used oral hypoglycaemic drugs other than metformin, if they used any investigational drug or had used a peroxisome proliferator activated-receptor $\gamma$ (PPAR $\gamma$ ) agonist within 4 months before the start of the study, or had a significant history of hypersensitivity to a peroxisome proliferator activated receptor $\gamma$ (PPAR $\gamma)$ agonist. Additional exclusion criteria were blood pressure exceeding 160/90 $\mathrm{mmHg}$, symptomatic postural hypotension, diuretic therapy for oedema, unstable or severe angina or congestive heart failure, any cardiovascular event in the last 6 months before entry to the study, the presence of clinically significant hepatic disease or anaemia, calculated creatinine clearance below $40 \mathrm{ml} / \mathrm{min}$, pregnancy, lactation, women of childbearing potential without appropriate contraception, and alcohol or drug abuse. To overcome confounding in the interpretation of the Ewing score, all participants using alpha or beta blockers were excluded. Study participants were either selected by advertisement or invited by their own physician at the outpatient clinic of the Radboud University Nijmegen Medical Centre or Rijnstate or Catharina Hospital. The participants received a payment and gave written informed consent. The study was approved by the hospital ethics committee, registered at clinical trials.gov and performed according to Good Clinical Practice guidelines.

\section{Procedure}

This was a randomised, placebo-controlled, double-blind, single-centre, parallel study with 4 weeks of single-blind run-in. At screening, the Ewing score was determined to quantify autonomic neuropathy [22]. In short, continuous finger arterial pressure and cardiac cycle duration (R-R interval) were recorded on a $\mathrm{PC}$-based data acquisition system during five standardised tests. Heart rate responses to the Valsalva manoeuvre (longest R-R interval after the manoeuvre divided by the shortest R-R interval during the manoeuvre; normal values are $>1.21$ ), to deep breathing (maximum-minimum heart rate during a breathing cycle; normal values $\geq 15 \mathrm{bpm}$ ) and to standing up (longest R-R interval $[ \pm 30$ th beat $]$ divided by the shortest $R-R$ interval $[ \pm 15$ th beat $]$; normal values are $\geq 1.04$ ) were measured to determine parasympathetic function, and the blood pressure responses to standing up (fall in systolic blood pressure [SBP]; normal values $\leq 10 \mathrm{mmHg}$ ) and to sustained hand grip (increase in diastolic blood pressure [DBP]; normal values $\geq 16 \mathrm{mmHg}$ ) were determined as a measure of sympathetic function. Each result was compared with the normal response and scored as normal, borderline or abnormal, which were allocated $0,1 / 2$ and 1 point respectively. The total score ranges from 0 to 5 . The participants were divided into two groups with Ewing score either $\geq 2.5$ or $<2.5$. Throughout this article the groups are referred to as having high versus low Ewing scores or established autonomic neuropathy versus mild or no autonomic neuropathy. Four weeks after screening (week -4 ), eligible participants were randomised to either rosiglitazone $4 \mathrm{mg}$ twice daily or placebo for 16 weeks in a 1:1 ratio (week 0 ) and balanced for the two Ewing score groups within $40-60 \%$ boundaries. The participants were assigned to study treatment in accordance with the randomisation schedule via the automatic GlaxoSmithKline Registration and Medication Ordering System, which could be reached by phone. At week 0 and week 16, primary endpoint experiments were performed. Vascular leakage was assessed as the transcapillary escape rate of albumin (TERalb). During all visits, including the control visits in weeks 4,8 and 12, adverse events and pill compliance were recorded and blood glucose lowering pharmacotherapy was adjusted. If a participant repeatedly had measurements below $4 \mathrm{mmol} / \mathrm{l}$, the metformin dose was decreased, as it was our intention to keep the insulin dose as constant as possible during the study. In addition, we performed a physical examination, measured body composition using bioimpedance and foot volume, and chemical, haematological, and glycaemic safety profiles were determined. There was a final follow-up visit in week 18. Participants were strictly advised to maintain their diet and not to change lifestyle throughout the study. 


\section{Experimental day}

Each participant attended the hospital after an overnight fast without taking insulin or oral blood glucose lowering pharmacotherapy in the morning. The procedure started at 08:00 hours in a quiet, temperature-controlled room $\left(23-24^{\circ} \mathrm{C}\right)$ with the participant in supine position. A venous catheter (Venflon, 20 gauge, $32 \mathrm{~mm}$; Becton Dickinson, Sandy, UT, USA) was inserted for the infusion of either insulin or glucose to keep the glucose level between 5 and $12 \mathrm{mmol} / \mathrm{l}$. During the experiment, plasma glucose was measured every 20 min (Glucocard Memory 2; Menarini, Florence, Italy). The participant was asked to inject his or her normal morning insulin dose. Then, a 20 gauge catheter (Angiocath; Becton Dickinson) was inserted into the left brachial artery under local anaesthesia $(0.3-0.4 \mathrm{ml}$ lidocaine $\mathrm{HCl} 20 \mathrm{mg} / \mathrm{ml}$; Braun, Melsungen, Germany), connected to an arterial pressure monitoring line and kept patent with heparin in saline $0.9 \%(2 \mathrm{U} / \mathrm{ml}, 3 \mathrm{ml} / \mathrm{h} ; \mathrm{NaCl} 0.9 \%$, Baxter, Utrecht, the Netherlands; heparin, Leo Pharma, Ballerup, Denmark). This catheter was used for blood sampling and blood pressure measurement. After $30 \mathrm{~min}$ of supine rest, blood was drawn for hormone analysis (atrial natriuretic peptide [ANP], aldosterone and renin) and for baseline TERalb measurement. An additional venous needle (Valu-Set, $0.6 \times 20 \mathrm{~mm}$; Becton Dickinson) was inserted and $7.4 \times 10^{4}-14.8 \times 10^{4} \mathrm{~Bq}^{125}$ I-labelled albumin (Schering Nederland, Weesp, the Netherlands) was given as an i.v. bolus injection at $0 \mathrm{~min}$. Over the next $60 \mathrm{~min}$, seven plasma samples were collected from the arterial line for radioactivity measurements.

\section{Bioimpedance}

During all visits, total body water (TBW) and extracellular volume (ECV) were assessed using an Akern 2000 bioelectrical impedance analyser [23] (Akern, Florence, Italy).

Foot volume

Foot volume was assessed by the water displacement method using an electronic balance (coefficient of variation, $0.30 \%$ ) [24]. The balance recorded the force necessary for a standardised immersion of the foot, which depends solely on the volume of the foot (Archimedes' principle).

Analytical methods

Plasma ANP was analysed by radioimmunoassay after cartridge extraction (Phoenix Pharmaceuticals, Burlingame, CA, USA). Plasma renin was determined with a two-site immunochemiluminometric assay (Diagnostic System Laboratories, Webster, TX, USA). Plasma aldosterone was analysed using antibody-coated tubes and competing radio- labelled aldosterone (Diagnostic Products, Los Angeles, CA, USA).

\section{Calculations}

For each TERalb test the measured radioactivity was plotted over time. An extinction curve was drawn assuming first-order kinetics. Slope and the extrapolated peak plasma concentration at $t=0$ were calculated using Microsoft Excel. Plasma volume and TERalb were calculated using the following formulas $[20,25]$ :

Plasma volume $(\mathrm{ml}) / 1.73 \mathrm{~m}^{2}=$

$(\mathrm{cpm}$ injected $/ \mathrm{cpm} t=0 / \mathrm{ml}) / \operatorname{surface}\left(\mathrm{m}^{2}\right) / 1.73 \mathrm{~m}^{2}$

TERalb $=\left(1-\mathrm{e}^{3600 \times \text { slope }}\right) \times 100(\% / \mathrm{h})$.

To ensure reliable results, calculated plasma volume and TERalb were excluded from primary endpoint analysis when the correlation coefficient between the extinction curve and the actual measured time points was below $80 \%$. Creatinine clearance was calculated with the Cockcroft formula [26].

\section{Statistical analysis}

The groups were balanced for sex and Ewing score. For variables measured at baseline and in week 16, only participants with paired observations were included. For variables with additional assessments, intention-to-treat populations both with and without last observation carried forward were analysed. The difference between the two treatment groups in either the total population or in one of the Ewing score subgroups was estimated by analysis of covariance with terms for treatment, sex, baseline measurement and, if applicable, Ewing score. Similarly, the difference in treatment effect between participants from different Ewing score subgroups was estimated by analysis of covariance with terms for sex, baseline and Ewing score. For the assessment of various relationships, the partial correlation coefficient was estimated with adjustment for sex. All significance tests were two-sided and the overall type I error was 5\%. Descriptive statistics are presented as mean and standard deviation or as percentage. Treatment effects are presented as mean with standard error. All statistical analyses were performed using the SAS software package (SAS Institute, Cary, NC, USA).

\section{Results}

A total of 40 participants were included in the study; 21 participants received placebo and 19 rosiglitazone (Fig. 2). One participant in the rosiglitazone group was withdrawn after 8 weeks because of atrial fibrillation and heart failure. Thirteen participants in the rosiglitazone-treated group and 




Fig. 2 Enrolment of study participants, distribution among subgroups and flow of participants through the study. ${ }^{\text {a }}$ To ensure reliable results, calculated transcapillary escape rates of albumin (TERalb) with correlation coefficients below $80 \%$ between the extinction curve and the actual measured time points were excluded from this analysis. FPG, fasting plasma glucose

17 participants in the placebo-treated group met the reliability criteria for TERalb measurements and were included in the analysis of the primary endpoint.

There were no clinically significant differences in baseline characteristics between the treatment groups in the total population, nor were there differences between the randomised subgroups, except for Ewing score (Table 1). Sixteen participants were classified with Ewing scores $\geq 2.5$.

Drug compliance was excellent. The 39 participants who finished the study reported only mild side effects. Oedema was more prevalent in the rosiglitazone group than in the placebo group ( $63 \%$ vs $24 \%, p<0.05)$, but always mild, and not different between participants with high and low Ewing scores.

\section{Total population}

In this section the model-adjusted treatment effects of rosiglitazone and placebo are given. A summary of the raw endpoint data at baseline and after treatment for the total population is provided in Electronic supplementary material (ESM) Table 1.
Effect of rosiglitazone on glycaemic control Rosiglitazone improved glycaemic control $\left(\mathrm{HbA}_{1 \mathrm{c}}\right.$; rosiglitazone $-0.67 \pm$ $0.15 \%$, placebo $-0.24 \pm 0.14 \%, p<0.05)$ despite a significant reduction in the daily insulin dose (rosiglitazone $-11 \pm 2 \mathrm{U} /$ day, placebo $+2 \pm 2 \mathrm{U} /$ day, $p<0.0001)$ and a slight decrease in background metformin treatment.

Effect of rosiglitazone on vascular leakage and diastolic blood pressure There was a trend for rosiglitazone to increase vascular leakage (TERalb; rosiglitazone $+1.38 \pm$ $0.46 \% / \mathrm{h}$, placebo $+0.29 \pm 0.40 \% / \mathrm{h}, p=0.09)$. Rosiglitazone decreased the intra-arterially measured DBP (rosiglitazone $-2.3 \pm 1.1 \mathrm{mmHg}$, placebo $+1.4 \pm 1.1 \mathrm{mmHg}, p=0.02$ ).

Effect of rosiglitazone on fluid variables and vascular hormones During rosiglitazone treatment, plasma volume (rosiglitazone $220 \pm 71 \mathrm{ml} / 1.73 \mathrm{~m}^{2}$, placebo $-21 \pm 64 \mathrm{ml} / 1.73 \mathrm{~m}^{2}, p=0.02$ ) and body weight (rosiglitazone $+2.4 \pm 0.5 \mathrm{~kg}$, placebo $+0.5 \pm 0.4 \mathrm{~kg}, p=0.004)$ increased, while haematocrit decreased (rosiglitazone $-0.024 \pm 0.005$, placebo $-0.005 \pm$ $0.005, p=0.007)$. Rosiglitazone did not increase ECV (rosiglitazone $+0.8 \pm 0.21$, placebo $+0.4 \pm 0.21, p=0.18$ ) and TBW (rosiglitazone $+1.0 \pm 0.31$, placebo $+0.4 \pm 0.31, p=0.12$ ), and had no effect on foot volume. In addition, rosiglitazone increased plasma ANP (rosiglitazone $+64 \pm 20 \mathrm{ng} / \mathrm{l}$, placebo $-1 \pm 18 \mathrm{ng} / \mathrm{l}, p=0.02$ ) but did not influence plasma renin and aldosterone levels.

\section{Low and high Ewing score subgroups}

A summary of raw endpoint data and model-adjusted treatment effects of rosiglitazone and placebo within these Ewing score subgroups is provided in Table 2.

Effect of rosiglitazone on glycaemic control The changes in glycaemic control and insulin requirements did not differ between the two Ewing score subgroups.

\section{Effect of rosiglitazone on vascular leakage and blood pressure} In the subgroup of patients with high Ewing scores, rosiglitazone significantly increased TERalb (rosiglitazone $2.43 \pm 0.45 \% / \mathrm{h}$, placebo $-0.11 \pm 0.42 \% / \mathrm{h}, p=0.002$ ), while rosiglitazone had no effect in the subgroup of patients with low Ewing scores (rosiglitazone $-0.44 \pm 0.72 \% / \mathrm{h}$, placebo $1.04 \pm 0.56 \% / \mathrm{h}$, not significant; Fig. 3a). As a result, rosiglitazone significantly increased TERalb in patients with high Ewing scores compared with those with low Ewing scores (high Ewing score $2.54 \pm 0.49 \% / \mathrm{h}$, low Ewing score $0.58 \pm 0.53 \% / \mathrm{h}, p=0.03$ ).

In the rosiglitazone-treated patients the reduction in intraarterial DBP tended to be more pronounced in participants with high Ewing scores (rosiglitazone $-3.1 \pm 1.2 \mathrm{mmHg}$, 
Table 1 Baseline characteristics

\begin{tabular}{|c|c|c|c|c|c|c|c|c|}
\hline \multirow[t]{2}{*}{ Variable } & \multicolumn{4}{|c|}{ Total population } & \multicolumn{2}{|c|}{ Ewing score $<2.5$} & \multicolumn{2}{|c|}{ Ewing score $\geq 2.5$} \\
\hline & $\begin{array}{l}\text { Rosiglitazone } \\
(n=19)\end{array}$ & $\begin{array}{l}\text { Placebo } \\
(n=21)\end{array}$ & $\begin{array}{l}\text { Ewing score } \\
<2.5(n=24)\end{array}$ & $\begin{array}{l}\text { Ewing } \\
\text { score } \geq 2.5 \\
(n=16)\end{array}$ & $\begin{array}{l}\text { Rosiglitazone } \\
(n=11)\end{array}$ & $\begin{array}{l}\text { Placebo } \\
(n=13)\end{array}$ & $\begin{array}{l}\text { Rosiglitazone } \\
(n=8)\end{array}$ & $\begin{array}{l}\text { Placebo } \\
(n=8)\end{array}$ \\
\hline Age (years) & $58 \pm 8$ & $59 \pm 10$ & $59 \pm 8$ & $58 \pm 10$ & $58 \pm 9$ & $60 \pm 8$ & $59 \pm 6$ & $57 \pm 13$ \\
\hline Male (\%) & 58 & 57 & 63 & 50 & 64 & 62 & 50 & 50 \\
\hline BMI $\left(\mathrm{kg} / \mathrm{m}^{2}\right)$ & $29 \pm 4$ & $29 \pm 6$ & $28 \pm 4$ & $31 \pm 6^{\mathrm{d}}$ & $28 \pm 4$ & $27 \pm 5$ & $30 \pm 3$ & $32 \pm 8$ \\
\hline $\begin{array}{l}\text { Waist circumference } \\
(\mathrm{cm})\end{array}$ & $101 \pm 10$ & $101 \pm 17$ & $97 \pm 13$ & $105 \pm 14$ & $98 \pm 10$ & $97 \pm 15$ & $104 \pm 9$ & $107 \pm 19$ \\
\hline SBP (mmHg) & $132 \pm 15$ & $132 \pm 15$ & $132 \pm 12$ & $132 \pm 18$ & $131 \pm 13$ & $133 \pm 13$ & $133 \pm 19$ & $131 \pm 19$ \\
\hline DBP (mmHg) & $80 \pm 6$ & $80 \pm 7$ & $80 \pm 6$ & $80 \pm 7$ & $80 \pm 4$ & $80 \pm 8$ & $79 \pm 8$ & $80 \pm 6$ \\
\hline Heart rate $(1 / \mathrm{min})$ & $73 \pm 10$ & $75 \pm 11$ & $73 \pm 12$ & $76 \pm 7$ & $70 \pm 10$ & $75 \pm 13$ & $77 \pm 7$ & $75 \pm 8$ \\
\hline $\begin{array}{l}\text { Calculated creatinine } \\
\text { clearance }(\mathrm{ml} / \mathrm{min})\end{array}$ & $89 \pm 16$ & $85 \pm 30$ & $86 \pm 19$ & $88 \pm 32$ & $92 \pm 15$ & $81 \pm 21$ & $84 \pm 17$ & $92 \pm 42$ \\
\hline $\begin{array}{l}\text { Urinary albumin/creatinine } \\
\text { ratio (mg/mmol } \\
\text { creatinine) }\end{array}$ & $0.9 \pm 1.4$ & $3.9 \pm 13.3$ & $0.9 \pm 1.3$ & $5.1 \pm 15.5$ & $1.0 \pm 1.9$ & $0.8 \pm 0.8$ & $0.9 \pm 0.5$ & $10.7 \pm 23.5$ \\
\hline $\begin{array}{l}\text { Duration of diabetes } \\
\text { (years) }\end{array}$ & $12.8 \pm 7.8$ & $14.1 \pm 5.7$ & $13.1 \pm 6.9$ & $14.0 \pm 6.5$ & $11.2 \pm 8.0$ & $14.6 \pm 5.8$ & $14.9 \pm 7.4$ & $13.1 \pm 5.9$ \\
\hline $\mathrm{HbA}_{1 \mathrm{c}}(\%)$ & $7.7 \pm 1.4$ & $8.1 \pm 1.1$ & $7.7 \pm 1.2$ & $8.3 \pm 1.3$ & $7.5 \pm 1.3$ & $7.8 \pm 1.0$ & $8.0 \pm 1.4$ & $8.6 \pm 1.1$ \\
\hline Insulin dose (U/day) & $60 \pm 40$ & $64 \pm 33$ & $58 \pm 28$ & $69 \pm 45$ & $57 \pm 24$ & $58 \pm 32$ & $64 \pm 56$ & $74 \pm 34$ \\
\hline Metformin (\%) & 32 & 43 & 29 & 50 & 18 & 38 & 50 & 50 \\
\hline Ewing score & $2.1 \pm 1.3$ & $1.7 \pm 0.9$ & $1.2 \pm 0.6$ & $3.0 \pm 0.8^{\mathrm{c}}$ & $1.2 \pm 0.6$ & $1.1 \pm 0.6$ & $3.3 \pm 0.9^{\mathrm{a}}$ & $2.7 \pm 0.5^{\mathrm{b}}$ \\
\hline
\end{tabular}

Data are mean $\pm \mathrm{SD}$

${ }^{\mathrm{a}} p<0.05$ versus rosiglitazone, Ewing score $<2.5$

${ }^{\mathrm{b}} p<0.05$ versus placebo, Ewing score $<2.5$

${ }^{\mathrm{c}} p<0.001$ versus Ewing score $<2.5$

${ }^{\mathrm{d}} p<0.05$ versus Ewing score $<2.5$

placebo $+1.2 \pm 1.3 \mathrm{mmHg}, p=0.03$ ) than in those with low Ewing scores (rosiglitazone $-2.2 \pm 1.7 \mathrm{mmHg}$, placebo $+1.6 \pm 1.5 \mathrm{mmHg}, p=0.11)$.

Effect of rosiglitazone on fluid variables and vascular hormones In the subgroup of patients with a high Ewing score, rosiglitazone did not increase fluid variables and decrease haematocrit significantly, while in the patients with low Ewing scores rosiglitazone seemed to increase plasma volume, TBW and body weight, and did decrease haematocrit (Table 2). The difference in effect of rosiglitazone over placebo in both subgroups was partly driven by the different response to placebo in these subgroups.

\section{Correlations}

Baseline Ewing score and change in vascular leakage or fluid variables In patients randomised to rosiglitazone, the increase in TERalb was highly correlated with the Ewing score at baseline ( $r=0.65, p=0.02$; Fig. $3 b)$, while the change in TERalb during placebo treatment was not $(r=0.12$, $p=0.66$ ). The correlation between the increase in TERalb during rosiglitazone treatment and baseline Ewing score was even more robust after inclusion of all the TERalb results $(r=0.72, p=0.002)$, showing that the exclusion of qualitative weak TERalb measurements neither biased nor caused the relationship. As participants with a high Ewing score had a significantly higher BMI than those with a low Ewing score, we also evaluated the correlation between the change in TERalb during rosiglitazone treatment and baseline BMI $(r=0.11)$ but this correlation was not significant. Furthermore, the changes in intra-arterial DBP while taking rosiglitazone were not significantly correlated with baseline Ewing score $(r=-0.28)$. In addition, the changes in body weight, haematocrit, plasma volume, TBW and ECV during rosiglitazone treatment were not correlated with the Ewing score at baseline.

Correlation between change in vascular leakage and change in diastolic blood pressure or fluid variables As expected from the vascular hypothesis, changes in DBP in patients receiving rosiglitazone were strongly inversely correlated to changes in vascular leakage in participants with high Ewing scores $(r=-0.96, p=0.002$, Fig. 4a), indicating that participants with a large decrease in blood pressure had a large increase in vascular leakage. This 


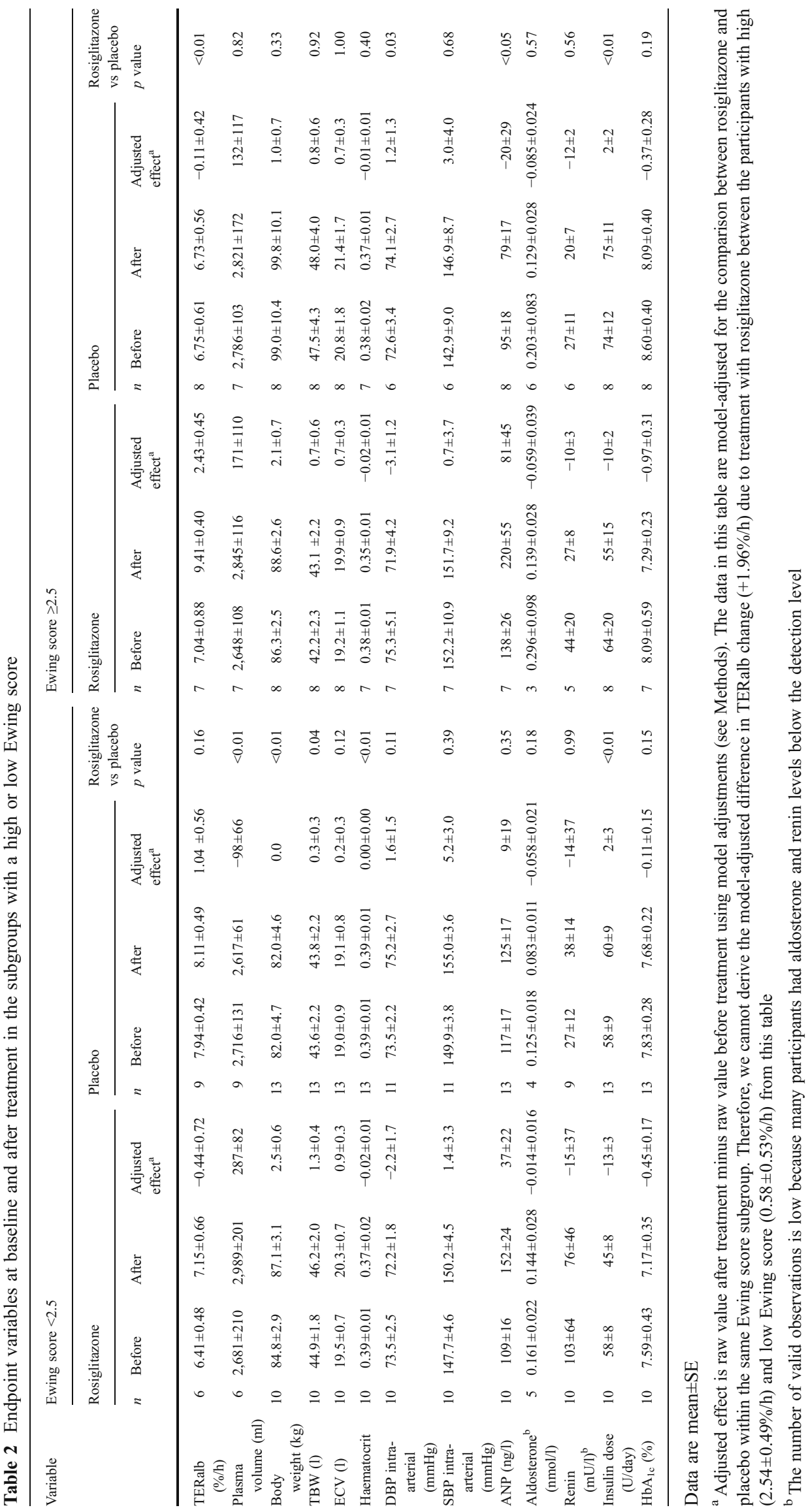




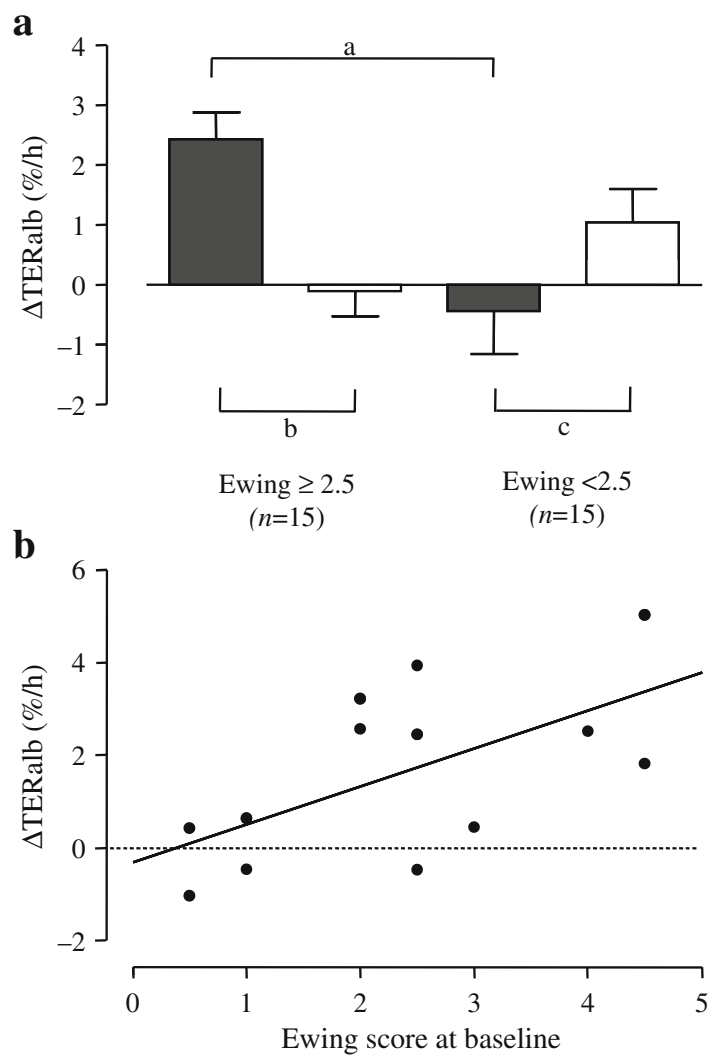

Fig. 3 Rosiglitazone induced vascular leakage in the autonomic neuropathy subgroups. a Mean model-adjusted change in transcapillary escape rate of albumin (TERalb) during treatment with either rosiglitazone or placebo within the subgroup of participants with a high Ewing score (left) and with a low Ewing score (right) (dichotomous). Values are means with SE. The data in this figure are model-adjusted for the comparison between rosiglitazone and placebo within a Ewing score subgroup. Therefore, we cannot derive from this figure the exact model-adjusted difference in TERalb change $(+1.96 \% / \mathrm{h})$ due to treatment with rosiglitazone between participants with high and low Ewing score (see Methods). Black bars, rosiglitazone; white bars, placebo. ${ }^{\mathrm{a}} p=0.002 ;{ }^{\mathrm{b}} p=0.03 ;{ }^{\mathrm{c}} \mathrm{NS}$. b Correlation $(r=0.65, p=0.02)$ between baseline Ewing score (continuous) and change in TERalb (individual raw data, therefore not model-adjusted) during treatment with rosiglitazone

correlation was not significant in participants with low Ewing scores.

In patients taking rosiglitazone, the change in vascular leakage was inversely correlated with the change in TBW ( $r=-0.76, p=0.004$; Fig. $4 b)$ and $\operatorname{ECV~}(r=-0.65$, $p=0.02)$.

\section{Discussion}

There are two main clinically relevant findings in the present study. First, in the presence of autonomic neuropathy, rosiglitazone induced vascular leakage in insulintreated patients with type 2 diabetes. Second, neither autonomic neuropathy nor the increase in vascular leakage in itself led to increased fluid retention. Together, these findings suggest that, in established autonomic neuropathy, thiazolidinediones will lead to exaggerated vascular leakage but not necessarily to more pronounced fluid retention. Nevertheless, increased vascular leak will render these patients more susceptible to oedema formation.

We postulate that thiazolidinediones have a vasodilator action, which subsequently promotes vascular leakage into interstitial tissues. In the present study, vascular leakage indeed tended to increase during treatment with rosiglitazone, although the effect was not statistically significant. Predefined subgroup analyses, however, showed a clear increase in vascular leak following treatment with rosiglitazone in participants with established autonomic neuropathy but not in participants with absent or mild autonomic neuropathy. This was confirmed by the positive correlation between Ewing score and change in TERalb. The findings
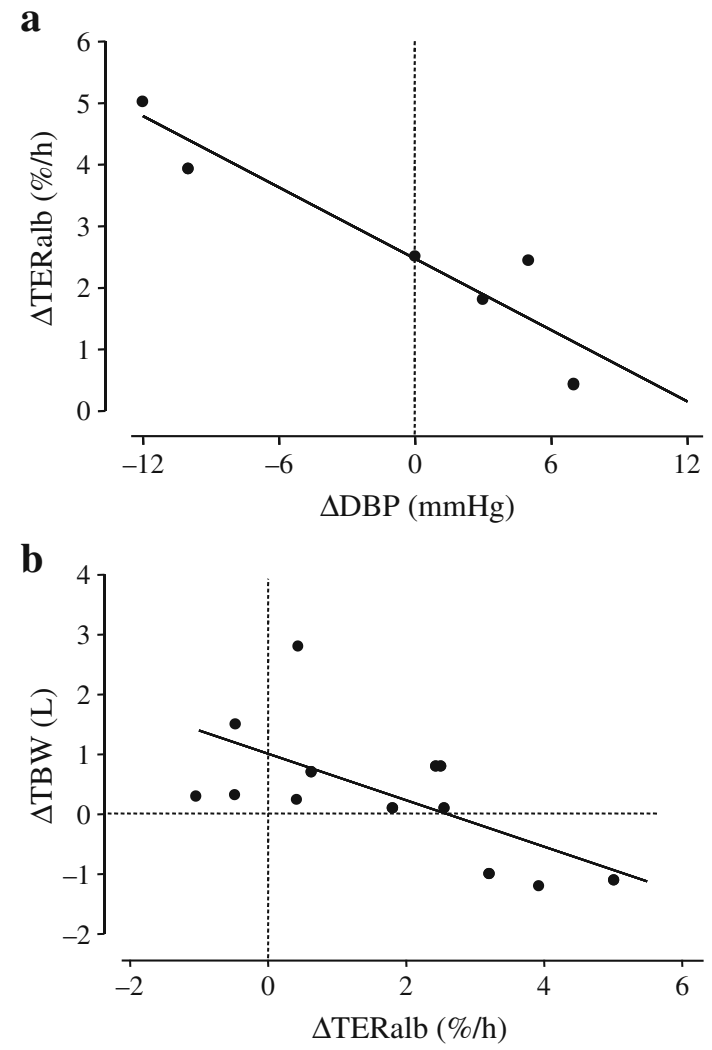

Fig. 4 Relationships of vascular leakage with diastolic blood pressure and total body water. a Inverse correlation $(r=-0.96, p=0.002)$ between rosiglitazone-induced changes in diastolic blood pressure (DBP) and transcapillary escape rate of albumin (TERalb) in participants on rosiglitazone with established autonomic neuropathy ( $n=7$; two participants had almost exactly the same readings for change in DBP and change in TERalb). b Inverse relationship ( $r=-0.76, p=0.004)$ between rosiglitazone-induced changes in TERalb and changes in total body water (TBW) in all participants treated with rosiglitazone with reliable TERalb measurements $(n=13)$ 
suggest that autonomic nerve damage in diabetic people prevents sympathetic nerve stimulation from counteracting the vasodilator effects of rosiglitazone. This concept (Fig. 1) is supported by the strong inverse correlation between changes in diastole. All in all, the findings fit with the concept that defective counter-regulation of haemodynamic changes caused by autonomic neuropathy exaggerates vascular leakage induced by thiazolidinediones. In persons with no or mild autonomic neuropathy, rosiglitazone did not increase vascular leakage, which is in accordance with previous human [20] and preclinical [27] findings.

The notion that autonomic neuropathy results in a defective counterbalance towards rosiglitazone-induced vasodilatation and subsequent vascular leakage has two consequences. First, people who are prone to vascular leakage should be protected against excessive plasma volume expansion, because excess fluid would leak out of the plasma compartment. Indeed, we did not observe either a disproportional increase in plasma volume or a decrease in haematocrit during rosiglitazone treatment in participants with established autonomic neuropathy. Second, excessive leakage should result in increases in TBW and ECV. This, however, was not observed in the present study: if anything, changes in TBW and ECV were smaller in the established autonomic neuropathy group. This apparent discrepancy can be explained by the complicated relation between sympathetic counter-regulation and renal sodium retention. The sympathetic nervous system responds to systemic hypoperfusion, both by direct renal sodium retention and by activation of the renin-angiotensinaldosterone system [28]. In fact, after drug-induced vasodilatation an intact sympathetic nervous system protects the body against local vascular leakage by reflex vasoconstriction and against systemic hypoperfusion by sodium retention. Consequently, a defective sympathetic nervous system will lead to vascular leakage without much sodium retention (Fig. 1). In both situations vascular leakage should be inversely correlated with and ECV and TBW, and this indeed is in complete agreement with our findings (Fig. 4b). The clinical implication of our observations is that insulin-treated patients with diabetic autonomic neuropathy may be partly protected from fluid overload by sodium retention induced by thiazolidinediones, but on the other hand will be more prone to vascular oedema, as a consequence of microvascular imbalance.

The prevalence of oedema was much higher in our study than in another study in insulin-treated patients performed by Raskin et al. [13], both during placebo treatment (present study 24\%, Raskin et al. 4\%) and during rosiglitazone treatment (present study 64\%, Raskin et al. $17 \%$ ). A potential explanation of this difference is that we were more focused on the development of oedema because oedema was a main outcome in our study. Another explanation could be the high prevalence of autonomic neuropathy in our population, although there was no correlation between Ewing score and the clinical finding of oedema. The reason we found differences in vascular leakage but not in oedema formation between the autonomic neuropathy subgroups during rosiglitazone treatment seems to be the absence of a reliable clinical test to quantitatively assess changes in total body interstitial fluid (oedema) in a chronic setting. Vascular leakage was measured as the mean of total body capillary leakage while oedema was measured only locally in the foot. For instance, changes in visceral vascular leakage will not influence foot volume.

In the present study, the glycaemic effect of rosiglitazone was moderate (a decrease in $\mathrm{HbA}_{1 \mathrm{c}}$ of $0.42 \%$ ) and seemingly less than expected. For example, Raskin et al. [13] reported a treatment effect of $-1.3 \%$. This difference may be explained by the shorter treatment period, the lower baseline $\mathrm{HbA}_{1 \mathrm{c}}$, the reduction in insulin dose, the slight decrease in background metformin use during rosiglitazone, and the marked glycaemic improvements in the placebo group.

The participant who was withdrawn from this study with atrial fibrillation had used beta blocker therapy for subjective palpitations in the past. She discontinued this therapy 1 month before entry into the study. Atrial fibrillation and oedema resolved quickly after reinstitution of beta blocker therapy and discontinuation of rosiglitazone. Despite correction of the rhythm, the participant was withdrawn as beta blocker therapy would have been a confounder in the final analyses.

To assess the degree of autonomic neuropathy, different methods, each with important limitations, are being used. We used the Ewing score, as it is well validated, widely accepted, non-invasive and suitable for screening. A disadvantage of the Ewing score is that there is no international consensus on how to adjust the tests for ageing. We have our own age-adjusted reference tables but these have not been published. Therefore, in this study we used the internationally accepted Ewing tests without age adjustment. We did perform a post hoc analysis after adjusting the Ewing score for age, but this did not result in any change in study outcome (data not shown).

We also measured baseline arterial plasma noradrenaline (norepinephrine) and adrenaline (epinephrine) and calculated the noradrenaline appearance rate (data not shown), which tended to be lower in the group with established autonomic neuropathy, suggesting that the higher Ewing score did indeed reflect autonomic neuropathy.

While sympathetic dysfunction is most relevant for our hypothesis, the Ewing score is based on parasympathetic as well as sympathetic tests [22]. In most 
patients the initial manifestation of autonomic disease is an abnormal response to the parasympathetic tests, followed by abnormal sympathetic tests in more severe autonomic neuropathy [21]. The increased frequency of abnormalities found with the parasympathetic tests may reflect both earlier involvement of parasympathetic damage and better sensitivity of the parasympathetic tests. To maximise the separation of participants with and without sympathetic neuropathy, we divided our population on the basis of the Ewing score into two categories with a cut-off value of 2.5, rather than the original four categories described by Ewing (normal, early, definite and severe). Indeed, more participants in the $\geq 2.5$ group had abnormalities in the sympathetic nerve tests than in the $<2.5$ group (data not shown). In line with the concept of sympathetic counterbalance, rosiglitazone increased vascular leakage in participants in whom a high Ewing score was combined with sympathetic disturbances in a post hoc analysis. On the contrary, rosiglitazone did not influence vascular leakage in participants with a low Ewing score and no sympathetic disturbances.

In conclusion, in insulin-treated type 2 diabetes patients who have autonomic neuropathy, thiazolidinediones increase vascular leakage and render the patient susceptible to the development of oedema. Autonomic neuropathy in itself does not exaggerate thiazolidinedione-induced fluid retention. Therefore, autonomic neuropathy should be considered as a risk factor for thiazolidinedione-induced oedema, not for fluid retention.

Acknowledgements This study was supported by GlaxoSmithKline. We thank the Department of Nephrology, Radboud Univeristy Nijmegen Medical Centre, for the use of the research room and the foot volume equipment. We thank $\mathrm{H}$. de Boer and B. Bravenboer, diabetologists at Rijnstate and Catherine Hospital, respectively, for their help in recruiting study participants.

Duality of interest M. W. Stewart is a full-time employee of GlaxoSmithKline. C. J. Tack and A. J. Rennings have participated in advisory panels for GlaxoSmithKline.

Open Access This article is distributed under the terms of the Creative Commons Attribution Noncommercial License which permits any noncommercial use, distribution, and reproduction in any medium, provided the original author(s) and source are credited.

\section{References}

1. Yki-Jarvinen H (2004) Thiazolidinediones. N Engl J Med 351:1106-1118

2. Kahn SE, Haffner SM, Heise MA et al (2006) Glycemic durability of rosiglitazone, metformin, or glyburide monotherapy. N Engl J Med 355:2427-2443

3. Parulkar AA, Pendergrass ML, Granda-Ayala R, Lee TR, Fonseca VA (2001) Nonhypoglycemic effects of thiazolidinediones. Ann Intern Med 134:61-71
4. Nissen SE, Wolski K (2007) Effect of rosiglitazone on the risk of myocardial infarction and death from cardiovascular causes. N Engl J Med 356:2457-2471

5. Home PD, Pocock SJ, Beck-Nielsen H et al (2007) Rosiglitazone evaluated for cardiovascular outcomes - an interim analysis. N Engl J Med 357:28-38

6. Gerstein HC, Miller ME, Byington RP et al (2008) Effects of intensive glucose lowering in type 2 diabetes. N Engl J Med 358:2545-2559

7. Home PD, Pocock SJ, Beck-Nielsen H et al (2009) Rosiglitazone evaluated for cardiovascular outcomes in oral agent combination therapy for type 2 diabetes (RECORD): a multicentre, randomised, open-label trial. Lancet 373:2125-2135

8. Dormandy JA, Charbonnel B, Eckland DJ et al (2005) Secondary prevention of macrovascular events in patients with type 2 diabetes in the PROactive Study (PROspective pioglitAzone Clinical Trial In macroVascular Events): a randomised controlled trial. Lancet 366:1279-1289

9. Nesto RW, Bell D, Bonow RO et al (2003) Thiazolidinedione use, fluid retention, and congestive heart failure: a consensus statement from the American Heart Association and American Diabetes Association. October 7, 2003. Circulation 108:2941-2948

10. Guan Y, Hao C, Cha DR et al (2005) Thiazolidinediones expand body fluid volume through PPARgamma stimulation of ENaCmediated renal salt absorption. Nat Med 11:861-866

11. Hong G, Lockhart A, Davis B et al (2003) PPARgamma activation enhances cell surface ENaCalpha via up-regulation of SGK1 in human collecting duct cells. FASEB J 17:1966-1968

12. Vallon V, Hummler E, Rieg T et al (2009) Thiazolidinedioneinduced fluid retention is independent of collecting duct alphaE$\mathrm{NaC}$ activity. J Am Soc Nephrol 20:721-729

13. Raskin P, Rendell M, Riddle MC, Dole JF, Freed MI, Rosenstock J (2001) A randomized trial of rosiglitazone therapy in patients with inadequately controlled insulin-treated type 2 diabetes. Diab Care 24:1226-1232

14. Shimkets RA, Warnock DG, Bositis CM et al (1994) Liddle's syndrome: heritable human hypertension caused by mutations in the beta subunit of the epithelial sodium channel. Cell 79:407-414

15. Shargorodsky M, Wainstein J, Gavish D, Leibovitz E, Matas Z, Zimlichman R (2003) Treatment with rosiglitazone reduces hyperinsulinemia and improves arterial elasticity in patients with type 2 diabetes mellitus. Am J Hypertens 16:617-622

16. Lindqvist M, Kahan T, Melcher A, Hjemdahl P (1994) Acute and chronic calcium antagonist treatment elevates sympathetic activity in primary hypertension. Hypertension 24:287-296

17. Page MM, Watkins PJ (1976) Provocation of postural hypotension by insulin in diabetic autonomic neuropathy. Diabetes 25:90-95

18. Seyer-Hansen K (1977) Postprandial hypotension. Br Med J 2:1262

19. Hilsted J, Frandsen H, Christensen NJ, Nielsen SL (1991) Plasma volume changes during hypoglycaemia: the effect of autonomic blockade. Eur J Clin Invest 21:22-26

20. Rennings AJ, Smits P, Stewart MW, Tack CJ (2006) Fluid retention and vascular effects of rosiglitazone in obese, insulinresistant, nondiabetic subjects. Diab Care 29:581-587

21. Vinik AI, Maser RE, Mitchell BD, Freeman R (2003) Diabetic autonomic neuropathy. Diab Care 26:1553-1579

22. Ewing DJ, Martyn CN, Young RJ, Clarke BF (1985) The value of cardiovascular autonomic function tests: 10 years experience in diabetes. Diab Care 8:491-498

23. Lukaski HC (1987) Methods for the assessment of human body composition: traditional and new. Am J Clin Nutr 46:537-556

24. van Hamersvelt HW, Kloke HJ, de Jong DJ, Koene RA, Huysmans FT (1996) Oedema formation with the vasodilators nifedipine and diazoxide: direct local effect or sodium retention? J Hypertens 14:1041-1045

25. Parving HH, Gyntelberg F (1973) Albumin transcapillary escape rate and plasma volume during long-term beta-adrenergic 
blockade in essential hypertension. Scand J Clin Lab Invest $32: 105-110$

26. Cockcroft DW, Gault MH (1976) Prediction of creatinine clearance from serum creatinine. Nephron 16:31-41

27. Cosson E, Cohen-Boulakia F, Tarhzaoui K et al (2009) Capillary endothelial but not lymphatic function is restored under rosiglitazone in Zucker diabetic fatty rats. Microvasc Res 77:220-225

28. Osborn JL, Holdaas H, Thames MD, DiBona GF (1983) Renal adrenoceptor mediation of antinatriuretic and renin secretion responses to low frequency renal nerve stimulation in the dog. Circ Res 53:298-305 